\title{
Comment mieux aider les médecins de la relève à assumer des responsabilités de gestion?
}

\section{How can better support be provided to the next generation of physicians taking on management responsibilities?}

\author{
Christian VOIROL 1,3,6, Marie-Claude AUDÉTAT ${ }^{1,5,7}$, Marie-France PELLAND ${ }^{1,5}$, \\ Julie LAJEUNESSE ${ }^{1,5}$, Raynald GAREAU ${ }^{2,4}$, Réjean DUPLAIN ${ }^{1,2}$ \\ 1 Département de médecine de famille et de médecine d'urgence, Université de Montréal, Montréal, Québec, Canada \\ 2 Campus de l'Université de Montréal en Mauricie, Trois-Rivières, Québec, Canada \\ 3 Département de Psychologie, Université de Montréal, Montréal, Québec, Canada \\ 4 Université du Québec à Trois-Rivières, Trois-Rivières, Québec, Canada \\ 5 Centre de Pédagogie Appliquée aux Sciences de la Santé (CPASS), Université de Montréal, Québec, Canada \\ 6 Haute École Arc Santé, Haute École Spécialisée de Suisse Occidentale, Neuchâtel, Suisse \\ 7 Unité de médecine de premier recours recherche et enseignement (UMPR), Faculté de médecine, Université de Genève, \\ Genève, Suisse
}

Manuscrit reçu le 8 juillet 2013 ; commentaires éditoriaux formulés aux auteurs le 30 septembre 2013 et le 4 octobre 2014 ; accepté pour publication le 6 octobre 2014

\author{
Mots-clés \\ Mobilisation ; \\ Relève ; Gestion ; \\ Compétences ; \\ Habiletés ; \\ Évaluation \\ de potentiel ; \\ Plan de carrière
}

\begin{abstract}
Résumé - Contexte : Depuis de nombreuses années et malgré une pléthore d'offres de formations en gestion et management, les facultés de médecine déplorent la difficulté à engager une relève médicale formée en gestion. Cet article veut analyser comment des médecins d'expérience en sont venus à assumer des responsabilités de gestion, quelles difficultés ils ont recontrées, quels moyens et ressources ils ont du mobiliser pour assumer leurs nouvelles responsabilités, quelles auraient été les ressources ou moyens préalables dont ils auraient pu bénéficier et enfin, pourquoi ils n'avaient pas mobilisé ces ressources et ces moyens avant leur entrée en poste. Méthodes : Une recherche exploratoire utilisant une méthodologie qualitative a été menée. Un groupe de discussion focalisée réunissant six médecins d'expérience, occupant ou ayant occupé différents postes à responsabilité en gestion, a été conduit. Les questions posées portaient sur les motivations, les circonstances et les conditions les ayant conduits à assumer des responsabilités de leadership et de gestion. Une analyse thématique du verbatim a été réalisée via le logiciel Atlas.ti 7. Résultats : Les résultats de cette recherche mettent en évidence que la majorité de ces médecins d'expérience n'ont pas suivi un plan de carrière
\end{abstract}




\section{Keywords} Mobilization; New generation; Management; Competencies; Skills; Assessment of potential Career Plan

consciemment établi mais ont plutôt saisi des opportunités que d'autres leur ont proposées. De plus, si ces médecins ne se sont pas formés avant d'occuper des postes à responsabilités de gestion, c'est essentiellement parce qu'ils méconnaissaient les compétences requises ainsi que les habiletés et l'expertise dont ils disposaient pour occuper de tels postes. Conclusions : Accompagner précocement les médecins de la relève susceptibles d'assumer des responsabilités de gestion dans 1) l'identification des habiletés et compétences requises pour faire de la gestion, 2) une évaluation objective de leur potentiel et 3) le développement d'un plan de carrière incluant la participation à des formations en gestion, est un préalable essentiel à leur mobilisation.

\begin{abstract}
Introduction: For many years and despite a plethora of management training offers regarding, medical schools have found it difficult to fill the need for a new generation of professionals trained in medical management. This article aims to analyze how and why experienced physicians chose to take on management responsibilities, the difficulties they experienced, the means and resources they mobilized to take on their new responsibilities, the resources or means they could have benefited from before taking on their new responsibilities and lastly the reasons why didn't they mobilize such resources and means before assuming the management position. Method: The method involved conducting an exploratory research using qualitative methodology. A focus group was set up with six experienced physicians who were occupying or had occupied various management positions. Questions were asked regarding motivation, circumstances and conditions that led them to assume their leadership and management responsibilities. On the basis of the verbatim responses, a thematic analysis was carried out using Atlas.ti 7 software. Results: The results show that most of these professionals did not follow a predefined career path. They rather seized opportunities that presented themselves. In addition, the main reason why these physicians had not trained prior to taking on management positions was that they had not identified the required management skills as well as the abilities and expertise they had acquired to assume their management responsibilities. Conclusion: One of the prerequisites in motivating the next generation of medical managers is to offer them early support by specifying the skills needed for their chosen career path (including a training program) and their actual abilities and expertise.
\end{abstract}

\section{Introduction et problématique}

Que ce soit dans le monde académique ou dans le réseau de la santé québécois, les organisations peinent à recruter et mobiliser des médecins formés pour occuper des postes de gestion. En effet, même s'il existe actuellement une pression croissante pour que les médecins s'impliquent davantage dans la gestion et l'administration ${ }^{[1,2]}$, ces responsabilités de gestion sont souvent endossées par des médecins peu formés en gestion et dont l'expertise est essentiellement médicale. Or, ces responsabilités de gestion recouvrent un ensemble de tâches complexes, essentielles au bon fonctionnement d'une organisation, qui ne peuvent pas s'acquérir dans le cadre d'une formation brève de quelques jours. En effet, assumer des responsabilités de gestion consiste à exercer des tâches telles que, par exemple, « définir une mission, des objectifs stratégiques, fonctionnels et opérationnels, organiser, planifier, décider, déléguer, diriger, donner du feed-back, évaluer, sanctionner, conduire des réunions, gérer des conflits, négocier, fixer un cadre, établir des processus, etc. ${ }^{[3-6]}$. Les objectifs de la formation des médecins canadiens décrits dans le cadre des compétences CanMEDS ${ }^{[7]}$ et dans le cadre des compétences en leadership médical du Collège Royal ${ }^{[8]}$, intègrent des compétences de leadership et de gestion ${ }^{[9]}$. Il faut noter à cet égard que le «leadership » correspond « [...] à la capacité d'un individu à mobiliser un groupe en faveur de finalités diverses $[. ..]{ }^{[10]}{ }^{[10}$ et ne représente donc pas un équivalent de ce que nous entendons par 
responsabilités de gestion. Cependant, en dépit de ces intentions affichées, les quelques préalables enseignés restent très souvent insuffisants pour permettre à ces professionnels d'assumer avec aisance les responsabilités qui incombent à un gestionnaire. Or, comme le souligne Romano, un parcours académique de haut niveau ne garantit nullement le succès en termes de leadership ou de gestion ${ }^{[11]}$. Par ailleurs, la complexification croissante des structures organisationnelles, l'évolution vers des pratiques interprofessionnelles ainsi que l'implication et la participation croissante des patients et des proches, rendent la gestion des équipes et des situations cliniques de plus en plus complexe ${ }^{[12-14]}$. Il devient dès lors primordial que le médecin assumant des tâches de gestion sache identifier les différentes facettes qui sous-tendent son travail, qu'il en comprenne le fonctionnement et la dynamique, et qu'il puisse optimiser sa pratique clinique et de gestion $^{[15]}$. Dans ce sens, de nombreux articles relèvent la nécessité de développer les capacités organisationnelles des futurs médecins et de donner aux leaders en émergence des pistes qui leur permettent de gérer ces défis organisationnels ${ }^{[16-19]}$.

Conscientes de la nécessité de développer la relève et de renforcer les compétences en gestion des médecins destinés à occuper des postes à responsabilités, de nombreuses institutions ont développé des formations, comme en témoigne par exemple la vaste littérature traitant de la formation des médecins aux compétences de gestion ${ }^{[20-23]}$, au leadership ${ }^{[17-19,24-30]}$, à la recherche et à l'enseignement universitaire ${ }^{[31-33]}$, à la gestion de carrière $^{[34-40]}$, à l'interdisciplinarité ${ }^{411]}$ ou encore à la connaissance de soi ${ }^{[42-44]}$. Pourtant, malgré cette pléthore de possibilités de formation, les résultats ne semblent pas être au rendez-vous. En effet, il faut constater qu'il y a peu de réelle demande ou d'intérêt manifesté par les médecins pour se former à la gestion $^{[45]}$. De la même manière, les médecins semblent peu intéressés pour embrasser des carrières comportant une forte proportion de tâches de gestion. En effet, les cliniciens sont la plupart du temps satisfaits de leur statut clinique ou pédagogique. Ils acceptent généralement de participer à des projets, pour autant que ces derniers affectent le moins possible leurs activités cliniques, mais ils hésitent encore souvent à s'impliquer davantage dans des tâches de gestion. Différents motifs sont évoqués pour expliquer cet apparent non intérêt des médecins pour la gestion. Nous pouvons citer, par exemple, les contraintes des contextes cliniques qui ne favorisent pas la disponibilité pour les tâches de gestion, la quasi-inexistence de leviers incitatifs à prendre de telles responsabilités de gestion, et bien entendu, le fait que les médecins ont choisi une carrière médicale et clinique, et non une carrière en gestion. Il reste que cette situation pose un sérieux problème, non seulement au réseau de la santé, mais également aux médecins eux-mêmes, puisqu'elle influence le potentiel d'amélioration du fonctionnement du réseau dans lequel ils travaillent et la qualité des soins offerts à leur clientèle. Enfin, outre qu'elles attestent que le recrutement d'une relève médicale formée en gestion est compliqué, différentes recherches ont également mis en évidence qu'une proportion non négligeable de médecins talentueux à qui des postes à responsabilités avaient été confiés sans préparation initiale, se sont retrouvés confrontés à des situations difficiles voire insurmontables à gérer ${ }^{[46,47]}$. Nous avons donc voulu mieux comprendre les processus en jeu dans l'engagement des médecins dans des postes de gestion.

\section{Questions de recherche}

Les questions auxquelles ce travail veut répondre sont donc les suivantes :

1) Comment et pourquoi des médecins d'expérience en sont-ils venus à assumer des responsabilités de gestion?

2) Quelles sont les difficultés qu'ils/elles ont rencontrées dans la prise en charge de ces fonctions?

3) Quelles sont les moyens et ressources qu'ils/ elles ont mobilisés pour assumer leurs nouvelles fonctions?

4) Quelles auraient été les ressources ou moyens préalables dont ils auraient pu bénéficier?

5) Si ces ressources et moyens étaient disponibles, pourquoi ne les ont-ils pas mobilisés avant leur entrée en poste? 
Une revue de littérature préliminaire n'a pas permis de faire émerger des articles ou des ouvrages susceptibles de répondre aux questions énoncées. En l'absence de références, une recherche exploratoire a donc été conduite.

\section{Méthodes}

Considérant la nature exploratoire de notre recherche, nous avons choisi une méthodologie qualitative en utilisant la technique du groupe de discussion focalisée.

\section{Participants}

Les participants ont été choisis selon le principe de l'échantillonnage intentionnel (ou par choix raisonné) décrit par Morse ${ }^{[48]}$. En effet, en l'absence d'informations disponibles sur les caractéristiques de la population des médecins gestionnaires qui ont œuvré ou œuvrent encore dans le système de santé québécois et au sein de la faculté de médecine de l'Université de Montréal, nous avons renoncé à choisir un échantillon représentatif. Les critères d'inclusion retenus ont été les suivants : médecins de famille seniors avec au moins 15 ans de pratique, membres du département de médecine de famille et de médecine d'urgence de la faculté de médecine de l'Université de Montréal, occupant ou ayant occupé différents postes à responsabilités dans le milieu académique (directeur de programme, chef de département, vicedoyen, doyen, etc.), hospitalier (chef d'unité, chef de département, etc.) et/ou au niveau des organes professionnels (collège des médecins, etc.), et bénéficiant d'une importante crédibilité aux yeux de leurs pairs.

Au total, 10 médecins ont été identifiés comme répondants aux critères ci-dessus et susceptibles de participer au groupe de discussion focalisée. Malheureusement, quatre médecins ont décliné notre invitation au dernier moment par manque de disponibilité et, finalement, six médecins $(N=6$, 2 hommes, 4 femmes) ont participé à la recherche.

\section{Collecte des données}

La collecte des données a été réalisée en utilisant la technique du groupe de discussion focalisée. En effet, les chercheurs ont rapidement formulé l'hypothèse qu'un échange d'expérience entre pairs serait plus riche que des rencontres individuelles. Par ailleurs, nous avons également considéré qu'en entendant l'ensemble des participants simultanément, nous pourrions au besoin mettre en lumière, par exemple, des normes ou des attitudes implicites caractéristiques du contexte, qui favoriseraient notre compréhension de la problématique étudiée ${ }^{[49,50]}$.

Le groupe de discussion focalisée, d'une durée d'une heure, s'est tenu à l'Université de Montréal lors de la journée annuelle de développement professoral au printemps 2011. Il a été animé par deux des chercheurs, deux autres chercheurs étant observateurs.

Le guide d'entretien pour le groupe de discussion focalisée a été élaboré sous forme de questions (cf. tableau I), qui ont été préparées par le groupe de chercheurs en tenant compte de la revue de la littérature et de leur expérience de gestionnaire. Les questions 1 et 3 explorent l'intérêt des médecins participants pour la gestion et leur vécu de gestionnaires, et les questions 2 et 4 explorent les variables susceptibles d'influencer la mobilisation et le développement de la relève en gestion.

Les participants étaient par ailleurs invités à aborder toute question qu'ils jugeaient importante pour l'avancement de la réflexion. Les échanges ont été enregistrés, puis transcrits sous forme de verbatim.

\section{Analyse des données}

L'analyse du verbatim s'appuie sur la méthode de l'analyse thématique ${ }^{[51]}$. A défaut de disposer de thématiques explicites au début du travail (bien que les questions de recherche aient pu orienter la définition des thématiques utilisées), nous avons adopté une démarche de thématisation continue ${ }^{[52]}$, similaire à celle utilisée dans la démarche de la théorie ancrée. En effet, même si cette approche est plus longue qu'une approche fondée sur une thématique préalable, elle favorise une riche description de la problématique, tout en permettant d'accroître le niveau de 
Tableau I. Le guide d'entretien pour le groupe de discussion focalisée.

Guide d'entretien : groupe de discussion focalisée des médecins « senior »

Pourquoi l'avez-vous fait : Comment avez-vous été amenés à occuper des fonctions de gestion et de supervision ? Avez-vous été capable de faire des choix de carrière lucidement et en étant informé ou les événements se sont-ils plutôt imposés à vous?

Comment l'avez-vous fait : Quelles compétences vous ont permis d'être en contrôle / de maîtriser les responsabilités que vous avez eu à assumer? Avez-vous eu l'impression de travailler avec votre « gros bon sens » au mieux de vos compétences, ou vous êtes vous appuyés sur des bases théoriques? Si oui, lesquelles (bases/fondements théoriques ou auteurs sur lesquels vous vous êtes appuyés)?

Comment l'avez-vous vécu : Comment vous sentiez-vous par rapport à vos activités professionnelles ? Par exemple, avez-vous eu du plaisir dans vos activités professionnelles à ce moment-là ? Vous êtes vous senti épuisé (burn out) à un moment donné de votre carrière, comment cela s'est-il manifesté, à quoi l'attribueriez-vous aujourd'hui et comment auriez-vous pu prévenir cette situation?

De quel soutien auriez-vous eu besoin : Rétrospectivement, de quoi auriez-vous eu besoin en termes d'accompagnement opérationnel et de formation au début de votre carrière qui vous aurait aidé à mieux assumer vos responsabilités personnelles, familiales, administratives, de gestionnaire/de leader (la question ne porte PAS sur les responsabilités cliniques) ? Qu'est-ce que vous auriez voulu connaître de vous qui vous aurait aidé à mieux assumer ces responsabilités?

synthèse au fur et à mesure, et de réduire le nombre de thèmes émergents.

Le codage et les analyses ont été effectués à l'aide du logiciel Atlas.ti 7 par deux chercheurs et une agente de recherche selon la méthode de l'analyse thématique décrite par Braun ${ }^{[51]}$. Tous ont lu individuellement la transcription des données, puis comparé et discuté leurs codages respectifs jusqu'à l'obtention d'un consensus. Les résultats ont ensuite été discutés et validés avec le reste des chercheurs du groupe.

\section{Résultats}

Les résultats sont présentés en fonction des principaux thèmes émergeants ; les citations sont identifiées par un code se référant aux lignes du verbatim.

\section{Un choix de carrière déterminé par d'autres et un déroulement de carrière la plupart du temps soumis aux opportunités}

Tous les participants relèvent le fait que même s'ils ont occupé des postes importants en gestion hospitalière ou universitaire, ils n'ont pas euxmêmes vraiment choisi leur carrière. Ce sont toujours d'autres collègues qui les ont sollicités pour des postes. La suite de leur carrière s'est déroulée selon le même principe, en fonction d'opportunités, sans formation préalable, sans réelle élaboration de plan de carrière, et sans même qu'ils soient forcément intéressés par la gestion ou par des domaines connexes.

"C'est là que ça se complique, j'ai été pris à prendre de façon inopinée un peu des fonctions administratives, ce pourquoi j'étais strictement non seulement pas formé, mais à toute fin pratique j'avais pas vraiment un intérêt de base àce niveau-là ». P 120928-194

«Moi j'ai été là par opportunité, j’ai pris des opportunités, j'ai jamais eu de plan véritablement, de plan de carrière ». P 120928-189

"J'ai dit oui sans vraiment savoir exactement ce qui se passait avec ça ». P 120928-121

"C'est parce qu'il y a des vides à un moment donné qui se font quand on est dans les organisations, il y a des vides qui se font et c'est facile d'être happé...». P 120928-208 
"Après, ça s'est enchaîné, plein d'offres de poste (...) bon ben ok c'est correct on y va, on embarque,...». P 120928-127

Des facteurs de motivation personnels qui expliquent leur implication malgré une méconnaissance des tâches spécifiques de gestion, voire un non intérêt

Les raisons qui ont motivé leur acceptation de ces postes sont essentiellement des motivations personnelles (par exemple, le souhait d'en faire partie, d'être utile) qui n'ont pas grand chose à voir avec leur désir de faire de la gestion. Aucun participant ne mentionne avoir jamais eu des compétences en gestion ou un intérêt particulier pour le domaine dans son ensemble, ou pour certaines tâches plus spécifiques.

Les motivations évoquées sont davantage reliées à un intérêt pour un domaine de la médecine, à l'importance de faire partie d'une communauté ou à l'envie de faire avancer les choses. Par ailleurs, les participants légitiment leur décision en faisant référence à d'autres désirs professionnels, eux non plus pas spécifiquement liés à des tâches de gestion, tels que le souhait de participer à la vie académique ou de ne pas exercer exclusivement une pratique clinique.

"Alors d'emblée, je choisissais un domaine où je savais qu'il faudrait exercer un leadership parce que tout était à faire ». P 120928-94

"Alors ça s'est fait comme ça pour moi, c'est par cette volonté de vouloir contribuer à améliorer les choses ». P 120928-163

"Quand on parle de choix éclairé ou pas, ben en fait je trouvais que c'était un beau défi, puis à ce moment là je ne me questionnais pas trop si cela correspondait à ce que j'avais le goût de faire, à mon plan futur, un beau défi, alors on embarque! ». P 120928-140

"Oui il y avait quelque chose à bâtir et ça m'interpellait et je voulais créer quelque chose ». P 120928-100

"C'était clair dans ma tête que je ne voulais pas juste faire de la pratique ». P 120928-223
«Je savais que je voulais, j’aimais ça, la vie universitaire, contribuer à la production de connaissances, avoir l'impression d'être "where the action is » ». P 120928-227

Une seule participante exprime clairement son intérêt pour la gestion. Mais elle précise que cela s'est développé en cours de route.

"J'ai embarqué, j'ai aimé l'expérience de gestion que je découvrais à ce moment-là puis (...) j'ai fait le saut (..) et je pense que je ne me suis pas nécessairement beaucoup questionnée avant d'avoir pris ces rôles-là que je considérais plus comme des beaux défis, puis je me disais ben on y va, on élargit puis on voit ce qui se passe». P 120928-143

Une méconnaissance des exigences et des compétences requises

Lorsqu'ils évoquent leur prise de fonction, aucun des participants ne considère qu'il était réellement formé en gestion ou dans un domaine connexe. Aucun ne semblait avoir connaissance des cadres conceptuels ou des théories en usage dans le domaine de la gestion et du leadership. Aucun d'eux ne semblait avoir conscience des exigences requises pour assumer de tels postes. Enfin, les participants ne semblaient pas du tout identifier ou savoir s'ils maîtrisaient ou non les compétences et habiletés personnelles sur lesquelles ils auraient pu s'appuyer pour prendre ces responsabilités.

«Moi en fait j'en avais pas du tout de compétences de gestionnaire, on s'entend, à part mon gros bon sens puis de me dire de me baser sur ce que j'avais vu quand j'étais résident». P 120928-249

«Je me sentais absolument pas outillé pour faire ça et j'avais pas l'appétit pour le faire non plus ». P 120928-403

"C'est un peu par nécessité que j'ai appris un peu (...), mais bien honnêtement pas par intérêt, 
j'ai pas véritablement un intérêt de base par rapport à ça». P 120928-199

Une expérience majoritairement pénible au début, et une grande solitude

Les expériences décrites par les participants témoignent toutes d'une expérience douloureuse ; la plupart se sont retrouvés seuls et sans appui à devoir gérer des situations pour lesquelles ils n'étaient pas préparés. Tous font état de leurs difficultés et des répercussions affectives et même somatiques. Il est cependant possible de relever dans leurs propos une tendance à minimiser la situation.

«J'ai eu à me battre au début ». P 120928-198

«J'ai pas eu de support de personne, j'ai pas eu de support universitaire,... ce n'est plus ce qui se passe maintenant mais dans le temps j'étais tout seul avec moi-même ». P 120928-252

"A ce moment-là je faisais de grands constats qu'on était totalement abandonné ». P 120928325

"C'est pas simple, moi en tout cas, j'ai des choses sur lequel j'ai eu des insomnies... très clairement des mauvais rêves ». P 120928-374

«Plus tu montes dans la hiérarchie plus tu es tout seul en haut, puis la solitude est extrêmement pénible quand on est dans un processus de gestion et c'est pas évident ». P 120928-518

"Une petite tape sur l'épaule par une personne d'expérience m'aurait néanmoins sauvé de quelques ulcères d'estomac ». P 110512-06

Des stratégies de développement des compétences et de coping élaborées essentiellement de façon intuitive avec la perception qu'il faut se débrouiller seul

Une fois confrontés à la réalité de leur tâche de gestion, les médecins ont fait preuve de capacités d'adaptation et ont dû développer un certain nombre de compétences. Ce développement s'est fait majoritairement par des stratégies centrées sur l'action, ainsi que par observation et « osmose ». D'autres, encore, relatent avoir « transféré » leurs compétences de leur domaine d'expertise à celui de la gestion.

«J'ai appris sur le tas comme on dirait (...) mais c'est comme ça que ça s'est passé, j'ai pris l'expérience en travaillant ». P 120928-262

«J'ai appris au contact de l'administration de mon hôpital, donc en étant à des réunions de service ou à différentes tables (...) mais en étant dans ce milieu-là déjà je voyais du monde travailler des dossiers, ça me donnait des pistes ». P 120928-318

«J'ai beaucoup appris par osmose (...) je regardais toujours comment les gens faisaient, puis je me disais qu'est-ce que j'aime dans ça? Qu'estce que j'aime moins? Qu'est-ce que je pourrais reproduire? Qu'est-ce qui a été efficace dans cette réunion dans laquelle moi j'ai été dirigée et là je m'imprégnais de ça, je me bâtissais mes capacités de cette façon-là ». P 120928-365

"Je suis chercheure hein, c'est une job être chercheure, faire de la recherche il faut que tu sois organisé structuré, il faut que tu sois capable de faire un argument, de convaincre du monde qu'il faut qu'ils te donnent de l'argent pour faire quelque chose et je pense que c'était des compétences, j'ai pu faire un transfert de cette espèce de gymnastique ». P 120928-356

\section{Un coaching ou un mentorat par opportunité et proximité}

Certains ont cependant ressenti le besoin de se faire aider et conseiller. Dans ce cas, c'est essentiellement une forme de coaching ou de mentorat de proximité qui s'est mis en place, plutôt qu'une formation spécifique en gestion. Ce soutien s'est révélé pertinent et apprécié.

«Mon mari a été mon meilleur coach (...) il avait été gestionnaire, il me donnait les clés 
d'analyse (...), des pistes puis il m'a donné $d u$ reflet sur moi-même aussi ». P 120928-326

"Quand j'avais un problème, moi je pouvais échanger avec ma conjointe qui n'était pas plus outillée que moi ». P 120928-425

"J'ai souvent eu besoin d'avoir recours aux conseils de gens plus expérimentés. J'ai ainsi été aidée par ceux qui ont occupé mon poste avant moi ». P 110725-02

Une formation en gestion tardive ouvrant sur le développement d'une réflexivité sur la tâche et sur les compétences

Les médecins n'ont pas souhaité ou n'ont pas ressenti le besoin d'aller se former avant de prendre des responsabilités de gestion. Plusieurs ont cependant suivi par la suite des formations qu'ils ont appréciées. Pour la majorité, ces formations leur ont alors permis de développer une réflexivité sur leurs tâches et leur carrière.

"Les cours...je pense qu'il faut que le besoin soit ressenti ». P 120928-483

"Mais je pense que le bagage de quelques années, six ans à peu près, à patauger là-dedans, ça m'a probablement fait aborder la formation différemment ». P 120928-287

"C'est peut-être (...) la formation XXXX il y a un an et demi à peu près, en fait, qui m'a permis de réfléchir ...quelles étaient mes valeurs, quelles étaient mes aspirations et ce que je tenais vraiment à accomplir (...) et je pense que depuis ce temps-là, peut-être mes décisions ont été un petit peu plus ciblées, plus en cohérence finalement avec ce que je voulais vraiment faire ». P 120928-150

«Ca m'a rejoint mais tellement! (...) Ils nous projetaient constamment dans la réflexion sur les systèmes de santé, l'organisations de soins, les besoins en éducation donc on était projeté làdedans et on nous appelait à développer nos idées ». P 120928-177
Un regard rétrospectif sur leur carrière, qui leur fait voir les choses différemment

Les participants ont beaucoup à dire sur leur parcours a posteriori. Tous s'accordent pour relever l'importance d'être soutenu et formé, principalement en termes de connaissances sur le travail à effectuer, et de démarche réflexive sur leur processus personnel.

"Mais certainement une première chose c'est de bien connaître le job (...) c'est quoi les défis de ça (...) Y a-t-il quelqu'un qui peut me parler de ça avant de commencer?». P 120928-448

"Et ça je l'ai appris à mes dépens, c'est la réflexion sur soi. Qui je suis et comment je vais... ça va être quoi mes difficultés à moi là-dedans. Qu'est-ce que je vais trouver difficile? Puis je veux pas m'épuiser là-dedans puis comme je disais tout à l'heure, j'ai pas le goût de faire de l'insomnie. Qu'est-ce qui va m'aider? C'est quoi les outils que j'ai pour pouvoir avancer làdedans en sachant comment je suis et ce que j'appréhende?». P 120928-448

"En fait j’ai pas bénéficié de mentorat et je réalise comment ça aurait été utile ...Tellement utile ». P 120928-492

"Moi je le vois selon deux grandes dimensions. Il y a la dimension personnelle puis il y a la dimension, je dirais plus technique, c'est à dire qu'est-ce que tu as à faire dans ton job, puis c'est pas forcément la même personne qui peut faire les deux, alors je trouve que c'est très très différent, puis je trouve que la dimension personnelle il faut aller la chercher le plus vite possible parce que la structure de personnalité, qui tu es, ne changera pas fondamentalement. Plus vite tu le sais, plus vite tu peux faire avec, puis te développer». P 120928-494

"Je pense que ce qui fait croître les individus (...) c'est l'accompagnement de ces gens-là puis c'est le feedback continu par rapport à ça». P 120928-523 


\section{Discussion}

Les résultats de cette recherche exploratoire mettent en évidence que les médecins seniors interviewés ont développé leur leadership et assumé des tâches de gestion essentiellement en fonction d'opportunités qui leur ont été présentées par des collègues plutôt que par choix personnel. Leur motivations, bien que très présentes, n'étaient pas reliées à des compétences ou à des intérêts relatifs à la gestion, mais plutôt au souci de bien faire, de relever un nouveau défi et d'assumer sa part.

Par ailleurs, ils avaient généralement sous-estimé les exigences des postes envisagés. Or, cette sousestimation se fondait non seulement sur une méconnaissance des habiletés requises pour assumer leur rôle de gestion, mais également sur une évaluation inadéquate des habiletés réelles dont ils disposaient pour assumer les responsabilités envisagées. Ce n'est qu'une fois en place que ces médecins ont mesuré l'ampleur de la tâche qui les attendait et qu'ils ont dû trouver des moyens pour développer leurs compétences. La plupart de ces médecins témoignent d'expériences douloureuses. Certes, ils ont su développer dans l'urgence des stratégies d'ajustement ponctuelles, essentiellement sur un mode intuitif, en se faisant plus ou moins formellement coacher ou en regardant fonctionner leurs pairs. Mais ce n'est qu'ultérieurement que les formations suivies leur ont permis de préciser leurs besoins, de mener une réflexion sur leur carrière et finalement de faire des choix plus conscients. Tous ont déploré le fait que personne ne les aient interpellés précocement afin de leur permettre d'anticiper les obstacles et de se préparer adéquatement à assumer de telles responsabilités.

Ces résultats mettent en évidence qu'au-delà de la prise de conscience des éventuelles lacunes ou besoins de formation, il y a probablement aussi un besoin de déconstruire certaines croyances et stratégies (par exemple, assumer seul les situations complexes plutôt que les déléguer et gérer en équipe), qui peuvent se révéler inadéquates dans le contexte de la gestion. Or ces croyances et ces stratégies sont non seulement fondées sur des expériences positives dans le domaine de la médecine, mais elles sont également valorisées et perpetuées par des pairs et des mentors bénéficiant d'une grosse crédibilité. Il s'agit donc d'amener les médecins qui constituent la relève potentielle pour assumer des responsabilités de gestion, à réévaluer la pertinence des habiletés, compétences, stratégies, croyances, normes, etc. qu'ils mobilisent dans leur pratique médicale quotidienne et qui font d'eux des professionnels qui réussissent, à l'aune des pratiques gagnantes dans le domaine de la gestion. Nous pensons que cette réévaluation ne peut se faire qu'à partir du moment où les médecins concernés prennent conscience des compétences réelles que requiert la gestion, du fait qu'il s'agit là d'une nouvelle profession pour eux, qui fait référence à ses propres croyances, normes, modèles conceptuels, etc. et qu'ils vont devoir se former en conséquence.

Il nous paraît dès lors, qu' avant de vouloir former ces médecins à la gestion, il faut les accompagner dans une réflexion sur leurs motivations, leur plan de carrière, les aider à identifier leur forces et leurs faiblesses, à prendre conscience de leur fonctionnement tant cognitif, affectif qu'émotionnel, à tester leur capacité d'influence, à explorer leur relation au pouvoir et à l'autorité, ou encore à évaluer leurs impacts sur les autres. Parmi les interventions les plus populaires pour développer la réflexivité chez les futurs responsables et leaders, le coaching et le mentorat figurent en bonnes positions ${ }^{[53,54]}$. Dans tous les cas et à l'instar de ce que qui se fait en médecine clinique $^{[55,56]}$, une telle démarche devrait être conduite par des modèles de rôles en gestion, expérimentés et bénéficiant d'une crédibilité et d'une reconnaissance importante du milieu.

\section{Limites de la recherche}

L'approche qualitative utilisée dans notre recherche comporte certaines limites. Premièrement, sur le plan de la fiabilité, cette recherche a mobilisé six participants. Bien que les expériences rapportées lors du groupe de discussion focalisée soient très homogènes et cohérentes, nous ne pouvons affirmer que la saturation des données ait été atteinte. Par ailleurs, si une triangulation entre les chercheurs a été réalisée, aucune triangulation sur les méthodes ou sur les sources d'information n'a été prévue. 
Deuxièmement, sur le plan de la crédibilité, il n'y a pas eu de démarche de validation par les répondants eux-mêmes. Cependant, plusieurs des auteurs de la recherche sont eux-mêmes des médecins d'expérience, occupant ou ayant occupé des postes de gestion dans des contextes très similaires à ceux des participants. En ce sens, il existe une forte probabilité que l'interprétation des informations collectées lors du groupe de discussion focalisée soit pertinente en regard de la réalité exprimée par les participants.

Troisièmement, sur le plan de la transférabilité, nous devons convenir que même si notre échantillon présente une importante diversité quant aux différents rôles de gestion assumés ou ayant été assumés (responsable de programme, chef de département, vice-doyen, doyen, chef d'unité de soins, chef de clinique, directeur hospitalier, etc.) et quant aux différents contextes dans lesquels ces rôles ont été ou sont encore assumés (universités, hôpitaux, cliniques, ordres professionnels, etc.), il est en revanche homogène en ce qui concerne la provenance des participants : ils assument tous actuellement des responsabilités au sein du département de médecine de famille et de médecine d'urgence de l'Université de Montréal. Dès lors, cet échantillon se réfère probablement à des normes, et des cultures professionnelles et institutionnelles spécifiques. Concrètement, notre échantillon est constitué de médecins de famille québécois francophones, assumant ou ayant assumés des postes à responsabilités dans le système public de santé québécois ou au sein de l'Université de Montréal. En conséquence, une généralisation des conclusions demanderait d'autres recherches élargissant la provenance des participants.

\section{Conclusions et perspectives}

Dans cette recherche, nous voulions comprendre pourquoi et comment des médecins d'expérience se sont engagés dans des postes de gestion. En particulier face à l'importante demande actuelle, il s'agissait d'identifier les déterminants incitatifs mais aussi les freins à l'engagement des médecins dans des fonctions de gestion. Nous souhaitions également comprendre pourquoi les médecins qui, finalement, acceptent d'embrasser une carrière de gestion, ne font pratiquement pas appel à l'offre pléthorique de formations à la gestion disponible sur le marché, avant d'assumer de tels postes à responsabilités.

Nos résultats mettent en évidence que les médecins ayant participé à cette recherche ne s'étaient pas formés avant d'occuper des postes à responsabilités de gestion essentiellement parce que :

1) Le choix d'endosser une carrière impliquant des tâches de gestion n'était pas la conséquence d'une réflexion structurée autour d'un plan de carrière, mais beaucoup plus une décision spontanée visant à saisir une opportunité qui se présente. En ce sens, ils n'imaginaient pas qu'ils allaient « faire de la gestion » à court ou moyen terme.

2) La majorité d'entre eux ont endossé des responsabilités de gestion en ignorant largement quelles sont les compétences et habiletés requises pour occuper de tels postes. Ils ignoraient en quoi consiste «faire de la gestion».

3) L'évaluation qu'ils faisaient de leurs propres habiletés et compétences pour assumer de tels postes restait très intuitive et imprécise. Ils ignoraient s'ils étaient correctement outillés ou non pour « faire de la gestion».

Ces résultats suggèrent qu'il serait opportun de développer un programme de formation permettant d'accompagner précocement les médecins de la relève susceptibles d'assumer des responsabilités de gestion. Cependant, l'objectif de ce programme ne serait pas de former ces médecins à la gestion, mais de les accompagner dans la découverte des habiletés et compétences requises, de les soutenir dans une évaluation objective de leur potentiel et de les accompagner dans le développement d'un plan de carrière, incluant la participation à des formations en gestion.

\section{Contributions}

Christian Voirol a piloté la recherche. Tous les auteurs ont contribué à la rédaction des questions du groupe de discussion focalisée. Christian Voirol, Julie Lajeunesse et Réjean Duplain ont animé le 
groupe de discussion focalisée. Le codage et l'analyse des données ont été faits par Marie-Claude Audétat, Christian Voirol et une agente de recherche. Une première version de l'article a été rédigée par Christian Voirol et Marie-Claude Audétat et chacun des autres auteurs a apporté une contribution significative dans la relecture et la rédaction finale de l'article.

\section{Remerciements}

Les auteurs remercient le département de médecine de famille et de médecine d'urgence de l'Université de Montréal pour la subvention de recherche de la Chaire Sadkoh Besrour, ainsi que tous les médecins seniors qui ont participé à la recherche. Les auteurs remercient également la Haute École Spécialisées de Suisse Occidentale (HES-SO) pour la subvention de valorisation ayant permis la rédaction de cet article.

\section{Déclaration d'intérêts}

Aucun auteur ne déclare de conflit d'intérêt en lien avec le contenu de cet article.

\section{Approbation éthique}

Cette recherche exploratoire fait partie d'un projet de recherche approuvé par le Comité d'éthique de la recherche en santé (CERES) de l'Université de Montréal (certificat \#12-045-CERES-D) délivré le 24 mai 2012.

\section{Références}

1. Stoller J. Developing Physician-Leaders: A Call to Action. J Gen Intern Med 2009;24:876-8.

2. Ramanujam R, Rousseau DM. The challenges are organizational not just clinical. J Organ Behav 2006;27:811-27.

3. Aubert N, Amoureux G, Hoffmann-Hervé M, Lebouin-Gélabert C, Lujan C, Taillardat J. Diriger et motiver : Art et pratique du management ( $2^{\text {ème }}$ éd.) Paris : Editions d'Organisation, 2002.

4. Chalvin D. Autodiagnostic des styles de management à l'usage des cadres et des dirigeants : connaissance du problème, applications pratiques. Paris : Éditions ESF-Entreprise moderne d'édition ; Librairies techniques, 1985.

5. Mintzberg H. Structure et dynamique des organisations. Paris - Montréal : Éditions d'Organisation Agence d'Arc, 1982.

6. Mintzberg H. The Nature of Managerial Work. New York: Harper \& Row, 1973.

7. Frank JR (Réd.). Le Cadre de compétences CanMEDS 2005 pour les médecins. L'excellence des normes, des médecins et des soins. Ottawa : Le Collège royal des médecins et chirurgiens du Canada, 2005.

8. NHS Institute for Innovation and Improvement and Academy of Medical Royal Colleges. Medical Leadership Competency Framework (3rd edition). Coventry: NHS Institute for Innovation and Improvement, 2010.

9. Fernandez N, Dory V, Ste-Marie L-G, Chaput M, Charlin B, Boucher A. Varying conceptions of competence: an analysis of how health sciences educators define competence. Med Educ 2012;46:357-65.

10. Parent F, Jouquan J, Azzi A. Comprendre que le leadership n'est pas uniquement un trait de personnalité mais qu'il peut se construire dans le cadre d'un apprentissage situé et réflexif. In: Parent F, Jouquan J (Dir.). Penser la formation des professionnels de la santé : une perspective intégrative. Bruxelles: De Boeck Supérieur, 2013:351-7.

11. Romano M. Ready or not: talented, high-achieving physicians often come up short in the skills and other attributes needed to excel as CEO. Mod Healthc 2004;34:26-34.

12. Armstrong PW. A time for transformative leadership in academic health sciences. Clin Investig Med 2007;30:E127-32.

13. Souba W, Mauger D, Day D. Does agreement on institutional values and leadership issues between deans and surgery chairs predict their institutions' performance? Acad Med 2007;82:272-80.

14. Souba W. Perspective: A new model of leadership performance in health care. Acad Med 2011;86: 1241-52. 
15. Arweiler D, Noyeau É, Charlin B, Millette B, Hodges B. Le leadership comme facteur de changement dans le champ de la santé : rôle de l'éducation médicale. Pédagogie Médicale 2011;11:239-53.

16. Ciampa EJ, Hunt AA, Arneson KO, Mordes DA, Oldham WH, Woo KV, et al. A workshop on leadership for $\mathrm{MD} / \mathrm{PhD}$ students. Med Educ Online 2011;16:7075.

17. Schwartz RW, Pogge CR, Gillis SA, Holsinger JW. Programs for the Development of Physician Leaders: A Curricular Process in Its Infancy. Acad Med 2000;75:133-40.

18. Goldstein AO, Calleson D, Bearman R, Steiner BD, Frasier PY, Slatt L. Teaching Advanced Leadership Skills in Community Service (ALSCS) to Medical Students. Acad Med 2009;84:754-64.

19. Fairchild DG, Benjamin EM, Gifford DR, Huot SJ. Physician Leadership: Enhancing the Career Development of Academic Physician Administrators and Leaders. Acad Med 2004;79:214-8.

20. Adamson BJ, Cant R V, Atyeo JW. Implications for tertiary education: managerial competencies required of beginning practitioners in the health service sector. Med Teach 2001;23:198-204.

21. Gunderman R, Kanter SL. Perspective: Educating Physicians to Lead Hospitals. Acad Med 2009;84: 1348-51.

22. Bonetta L (Ed.). Making the Right Moves: A Practical Guide to Scientific Management for Postdocs and New Faculty. Chevy Chase : Howard Hughes Medical Institute and Burroughs Wellcome Fund, 2004.

23. Bouleau N. Martingales et marchés financiers. Paris: Ed. Odile Jacob, 1998.

24. Foster T, Regan-Smith M, Murray C, Dysinger W, Homa K, Johnson LM, et al. Residency Education, Preventive Medicine, and Population Health Care Improvement: The Dartmouth-Hitchcock Leadership Preventive Medicine Approach. Acad Med 2008;83:390-8.

25. Kahn LH. A Prescription For Change: The Need For Qualified Physician Leadership In Public Health. Health Aff 2003;22:241-8.

26. Crites GE, Ebert JR, Schuster RJ. Beyond the Dual Degree: Development of a Five-Year Program in Leadership for Medical Undergraduates. Acad Med 2008;83:52-8.
27. Ovretveit J. Improvement leaders: what do they and should they do? A summary of a review of research. Qual Saf Heal Care 2010;19:490-2.

28. Rusch LM. Supporting clinical nursing leadership and professional practice at the unit level. Nurs Leadersh Forum 2004;9:61-6.

29. Stuart H, Diane H. Leadership coaching in health care. Leadersh Organ Dev J 2006;27:183-9.

30. Warren OJ, Carnall R. Medical leadership: why it's important, what is required, and how we develop it. Postgrad Med J 2011;87:27-32.

31. Bateman H, Kinmonth AL. Journeys and pathways: exploring the role of professional development advice and educational guidance for practitioners expressing interest in research. Med Educ 2001; $35: 49-55$.

32. Von Glinow MA. The CCL Guide to Leadership in Action: How Managers and Organizations Can Improve the Practice of Leadership. Acad Manag Rev 2005;30:440-2.

33. Trevitt C, Perera C. Self and continuing professional learning (development): issues of curriculum and identity in developing academic practice. Teach High Educ 2009;14:347-59.

34. Kanter SL. Career Guidance and the Quality of the dialogue. Acad Med 2011;86:149-50.

35. Rabow MW, Remen RN, Parmelee DX, Inui TS. Professional Formation: Extending Medicine's Lineage of Service Into the Next Century. Acad Med 2010; 85:310-7.

36. Bogue RJ, Guarneri JG, Reed M, Bradley K, Hughes J, Bogue BRJ. Secrets of Physician Satisfaction: Study identifies pressure points and reveals life practices of highly satisfied doctors. Physician Exec 2006;32:30-8.

37. Sturges J, Conway N, Liefooghe A. Organizational Support, Individual Attributes, and the Practice of Career Self-Management Behavior. Gr Organ Manag 2010;35:108-41.

38. Newbold M. Guiding Doctors in Managing their Careers. Clin Manag 2006;14(3):169-70.

39. Mazmanian PE. Reflecting on practice, professional growth, and certification. J Contin Educ Health Prof 2007;27:141-2. 
40. Page D. "Most Improved" Take Similar Steps to Reach New Heights: Enthusiastic executives engage physicians and staff in IT decisions to improve quality of care and financial performance. Hosp Heal Networks 2011;85:42-4.

41. Larson EL. New rules for the game: Interdisciplinary education for health professionals. Nurs Outlook 1995;43:180-5.

42. Hiermann W, Hofferer M. A practical knowledge-based approach to skill management and personal development. J Univers Comput Sci 2003;9:1398-409.

43. Xu G, Paddock LE, O’Connor JP, Nash DB, Buehler ML, Bard M. Physician Executives Report High Job Satisfaction. Physician Exec 2001;27:46-7.

44. Wilkinson S, Stainsby K, Robbins M. Develop your interpersonal and self-management skills: A practical resource for healthcare administrative and clerical staff. Oxford (UK): Radcliffe, 2008.

45. Association québécoise d'établissements de santé et de services sociaux (AQESSS). Modèles et enjeux du partenariat médico-administratif: état des connaissances. Montréal, Canada: Association québécoise d'établissements de santé et de services sociaux. 2012 [On-line] Disponible sur : www.aqesss.qc.ca.

46. Prévost C, Roman S. Satisfaction professionnelle et conditions de pratique : deux éléments qui ont leur importance. Médecin du Québec. 2009;44:55.

47. Johnson C. Les périls du perfectionnisme ou comment devenir son propre ennemi. Médecin du Québec. 2009;44:37.

48. Morse J. Strategies of intraproject sampling. In: Munhall PL (Ed.) Nursing Research: A Qualitative
Perspective. Sudbrury (MA) : Jones and Bartlett, 2007:529-38.

49. Kitzinger J. The methodology of Focus Groups: the importance of interaction between research participants. Sociol Health Illn 1994;16:103-21.

50. Krueger RA, Casey MA. Focus Groups: A Practical Guide for Applied Research. Thousand Oaks (CA) : SAGE Publications, 2000.

51. Braun V, Clarke V. Using thematic analysis in psychology. Qual Res Psychol 2006;3:77-101.

52. Paillé P, Mucchielli A. L'analyse qualitative en sciences humaines et sociales ( $3^{\text {ème }}$ éd.) Paris : Armand Colin, 2012.

53. Coates WC. Being a Mentor: What's in It for Me? Acad Emerg Med 2012;19:92-7.

54. Sambunjak D, Straus S, Marusic A. A Systematic Review of Qualitative Research on the Meaning and Characteristics of Mentoring in Academic Medicine. J Gen Intern Med 2010;25:72-8.

55. Cruess SR, Cruess RL, Steinert Y. Role modelling making the most of a powerful teaching strategy. Br Med J 2008;336:718-21.

56. Côté L, Leclère H. How clinical teachers perceive the doctor-patient relationship and themselves as role models. Acad Med 2000;75:1117-24.

Correspondance et offprints : Christian Voirol, Université de Montréal, Québec, Canada et Haute école Arc Santé - Haute École Spécialisée de Suisse Occidentale (HES-SO), University of applied sciences of Western Switzerland. Espace de l'Europe 11, 2000 Neuchâtel, Suisse. Téléphone : +41 329301212 - Téléphone direct : +41329302554 - Télécopie : +41329301213.

Mailto : christian.voirol@he-arc.ch 